\title{
Racial disparities in breast cancer diagnosis in Central Georgia in the United States
}

\author{
Bilal Farooqi, MD, ,ab Betsy Smith, MPH, ${ }^{a}$ Mudit Chowdhary, BS, ${ }^{a}$ Susan Pavoni, BS, ${ }^{b}$ \\ Aadil Modi, MD, ${ }^{a}$ and Frederick Schnell, MD $^{\mathrm{a}, \mathrm{b}}$
}

${ }^{a}$ Department of Medicine, Mercer University School of Medicine; and ${ }^{\mathrm{b} C a n c e r}$ Life Center, Medical Center Navicent Health, Macon, Georgia

Background Mortality rates in breast cancer are worse for African Americans than for whites.

Objective To investigate the presence of racial disparities in clinical staging in women diagnosed with breast cancer and understand whether such disparities exist in Central Georgia in the United States.

Methods We retrospectively reviewed records from the Tumor Registry of the Medical Center Navicent Health in Macon, Georgia, of women who had been diagnosed with breast cancer during 2011-2013. The chi-square test was used to assess statistically significant differences between whites and African Americans. We also assessed the patients' health insurance status and age at diagnosis.

Results A total of 578 participants were identified. Statistically significant differences existed in the clinical stage between the races $(P=.0003)$. Whites were more often clinical stage I at diagnosis, whereas African Americans had a greater percentage of stages II, III, or IV. African Americans were more than twice as likely to be diagnosed at clinical stage IV than were their white counterparts. Statistical differences also existed with age at diagnosis $(P=.0066)$ and insurance coverage $(P=.0004)$. A greater percentage of white patients were aged 65 years or older at diagnosis, whereas a greater percentage of African American patients were aged 49 years or younger. A greater percentage of African Americans had Medicaid insurance, whereas a greater percentage of whites had private health insurance.

Limitations As a single-center study, it is difficult to generalize these results elsewhere. Furthermore, this study focused on association and not on causation. It is difficult to pinpoint why such disparities exist.

Conclusion The etiology of racial disparities between African American and white women with breast cancer seems to be multifaceted. Screening mammography remains an important tool for identifying breast cancer. Low socioeconomic and educational status as well as a lack of a primary care physician may play a role in these disparities. Other factors that may have a role include biological factors and possible mistrust of the health care system.

$\mathrm{B}$ reast cancer is the most common noncutaneous malignancy and the second most common cause of cancer mortality among women in the United States. ${ }^{1}$ Study findings have shown that breast cancer is more prevalent in white women than in African American women; however, mortality due to breast cancer is greater and more burdensome among African American women.,3 In 1977, the cancer mortality rate among African American and white women was almost equal, with African American women having a mortality rate of 32.8 per 100,000 of the population, compared with 32.7 per 100,000 in white women. However, since that time, a disparity between the races has emerged, ${ }^{4,5}$ with the mortality rate for breast cancer from 2008-2012 reported as 31.0 per 100,000 for
non-Hispanic African American women and 21.9 per 100,000 for non-Hispanic Caucasian women. ${ }^{6}$

The cause of documented increased mortality in African American women may prove multifaceted and complex. Possible etiologies of increasing racial disparities may include health insurance status, socioeconomic differences, availability of health care, diagnostic delays, biological factors, and personal beliefs. ${ }^{3-11}$ Although eradicating health disparities remains a national imperative, the mortality gap between African American and white women has increased only in regard to breast cancer. ${ }^{4}$ Therefore, we aimed to examine racial disparities in diagnosing breast cancer and gain a better understanding of whether such disparities exist in Central Georgia.

Accepted for publication September 21, 2015. Correspondence: Bilal Farooqi, MD; bilalfarooqi@gmail.com. Disclosures: Dr Schnell has held consultative arrangements with Celgene, Merck, Roche-Genentech, and Taiho. The other authors have no disclosures. JCSO 2015;13:436-441. (02015 Frontline Medical Communications. DOI 10.12788/jcso.0198. 


\section{Methods}

\section{Study design and population}

This retrospective cohort study used data from a tumor registry collected at a single institution, the Medical Center Navicent Health (MCNH), in Macon, Georgia. The center, which was formerly known as the Medical Center of Central Georgia, is an academic medical center affiliated with Mercer University School of Medicine and serves a primary and secondary service area of 30 counties with a population of nearly 750,000. It is the second largest hospital in Georgia and is licensed for more than 600 beds. The study was approved by the center's institutional review board.

The Cancer Life Center at MCNH, which has been accredited by the Commission on Cancer since 1992, sees close to 2,000 newly diagnosed or active cancer patients annually. It uses a tumor registry to collect data on all patients seen at any Navicent Health facility who are diagnosed with cancer or have active disease. The data are used internally as well as reported to the National Cancer Data Base (NCDB) of the Commission on Cancer and the Georgia Comprehensive Cancer Registry.

The tumor registry collects demographic information such as sex, race, age, insurance coverage, marital status, and county of diagnosis. Added clinical information collected can include medical history (physical findings, screening information, history of previous cancer); diagnostic findings (types, dates and results of procedures used to diagnose cancer); cancer information (primary site, cell type, extent of disease, grade, stage); cancer therapy (surgery, radiation therapy, chemotherapy, hormone, immunotherapy); and follow-up surveillance information (additional treatment, recurrence, patient status).

\section{Factors assessed}

Demographic information used for this analysis included age at diagnosis, race defined as white and African American, year of diagnosis, and insurance coverage defined as Medicaid, Medicare, private coverage, coverage for military personnel and veterans under the Veterans Affairs (Tricare/VA), and not insured or with unknown coverage. Clinical information used for this analysis included clinical stage group, pathologic stage group, and grade differentiation. Clinical stage and pathologic stage groups were defined as stages 0, I, II, III, and IV; grade differentiation was defined as I, II, and III. Men and patients with a race other than white or African American were excluded because of their small sample sizes within these categories. Patients diagnosed in 2011, 2012, and 2013 analyzed for this study to represent a sample size of 578 patients.

\section{Statistical analyses}

Descriptive statistics were reported as means and standard deviations for continuous variables and as frequencies and percentages for categorical variables. A chi-square test was used to determine if statistical differences existed between whites and African Americans. A $P$ value of .05 was used to determine statistical significance. SAS version 9.4 was used for all analyses (SAS Institute, Cary, NC).

\section{Results}

Demographic characteristics of the study population $(\mathrm{N}=578)$ are reported in Table 1 . Of the 578 participants, $60 \%$ were white and $40 \%$ were African American. There was a statistically significant difference in age at diagnosis $(P=.0066)$. About $40 \%$ of the patients were aged 50-64 years overall and by race, but a greater percentage of whites were aged 65 years or older at diagnosis compared with African Americans. Among African American women, 27\% were diagnosed when they were 35-49 years old, compared with $20 \%$ of white women; and 3.42\% African Americans were diagnosed when they were 18-34 years old, compared with $0.87 \%$ of whites. The mean age at diagnosis was 57.54 years (SD, 12.68) for African Americans, and 60.43 years (SD, 12.35) for whites. There was an even distribution of breast cancer diagnoses between the years examined. Most of the patients overall held either private insurance (47.92\%) or Medicare (33.22\%), but there was a statistical difference when comparing health insurance coverage between whites and African Americans $(P=$ .0004). A greater percentage of African Americans had Medicaid, whereas a greater percentage of whites had private insurance (Table 1, Figure 1). There was a similar distribution between the 2 races of patients who had Medicare and Tricare/VA coverage. A slightly greater percentage of whites were uninsured or had unknown coverage compared with African Americans.

\section{Staging results}

There was a statistically significant difference between white and African American patients in disease stage at the clinical diagnosis of breast cancer $(P=.0003$, Table 2$)$. Whites were more likely to present with clinical diagnosis of stage I breast cancer than were African Americans (49.68\% vs $32.26 \%$, respectively), whereas a greater percentage of African Americans were diagnosed at stages II, III, and IV (Table 2, Figure 2). More than twice as many African American women were diagnosed with clinical stage IV breast cancer compared with whites $(11.98 \%$ vs. 4.78\%). There was a statistical trend in pathologic stage diagnosis between whites and African Americans $(P=.05$, Table 2). A greater percentage of African Americans had a pathologic stage of III and IV than did whites. No statistically significant difference in grade differentiation between the 2 races existed in the study population. 
TABLE 1 Demographic characteristics of women diagnosed with breast cancer from the tumor registry of the Cancer Life Center at Navicent Health, Macon, Georgia, 201 1-2013

\begin{tabular}{|c|c|c|c|c|}
\hline Characteristic & $\begin{array}{c}\text { Overall, n (\%) } \\
{[N=578]}\end{array}$ & $\begin{array}{c}\text { White, n (\%) } \\
{[n=344]}\end{array}$ & $\begin{array}{c}\text { African } \\
\text { American, } \\
\text { n (\%) [n = } \\
234]\end{array}$ & P value* \\
\hline Age at diagnosis, y & & & & .0066 \\
\hline $\begin{array}{l}18-34 \\
35-49 \\
50-64 \\
65+\end{array}$ & $\begin{array}{c}11(1.90) \\
134(23.18) \\
239(41.35) \\
194(33.56)\end{array}$ & $\begin{array}{c}3(0.87) \\
70(20.35) \\
141(40.99) \\
130(37.79)\end{array}$ & $\begin{array}{c}8(3.42) \\
64(27.35) \\
98(41.88) \\
64(27.35)\end{array}$ & \\
\hline Year of diagnosis & & & & .15 \\
\hline $\begin{array}{l}2011 \\
2012 \\
2013\end{array}$ & $\begin{array}{l}201(34.78) \\
195(33.74) \\
182(31.49)\end{array}$ & $\begin{array}{l}118(34.30) \\
126(36.63) \\
100(29.07)\end{array}$ & $\begin{array}{l}83(35.47) \\
69(29.49) \\
82(35.04)\end{array}$ & \\
\hline Type of insurance & & & & .0004 \\
\hline $\begin{array}{l}\text { Medicaid } \\
\text { Medicare } \\
\text { Private } \\
\text { Tricare/VA } \\
\text { Not insured/unknown }\end{array}$ & $\begin{array}{c}86(14.88) \\
192(33.22) \\
277(47.92) \\
8(1.38) \\
15(2.60)\end{array}$ & $\begin{array}{c}33(9.59) \\
116(33.72) \\
179(52.03) \\
5(1.45) \\
11(3.20)\end{array}$ & $\begin{aligned} 53 & (22.65) \\
76 & (32.48) \\
98 & (41.88) \\
3 & (1.28) \\
4 & (1.71)\end{aligned}$ & \\
\hline
\end{tabular}

Tricare/VA, Tricare/Veterans Affairs, insurance for military personnel and veterans under the Veterans Affairs

*Derived from chi-square test.

multifactorial and complex. Various reasons have been ascribed to the increased mortality in African American women from such studies. Possible implicating factors can include socioeconomic as well as biological status. ${ }^{3}$ In our study, a greater percentage of African Americans were insured through Medicaid, whereas a greater percentage of whites had private health insurance. We did not determined patient socioeconomic status in our study, but these findings may imply that socioeconomic status was a factor. We will look to explore some of these possible etiologies in this paper.

\section{Screening mammography}

Screening mammography is a crucial resource in the early diagnosis of breast cancer. Randomized controlled trials have demonstrated that screening mammography can lead to a reduction in cancer mortality rates. ${ }^{12}$ Recommendations for screening mammography vary by professional institutions, but many organizations recommend annual screen-

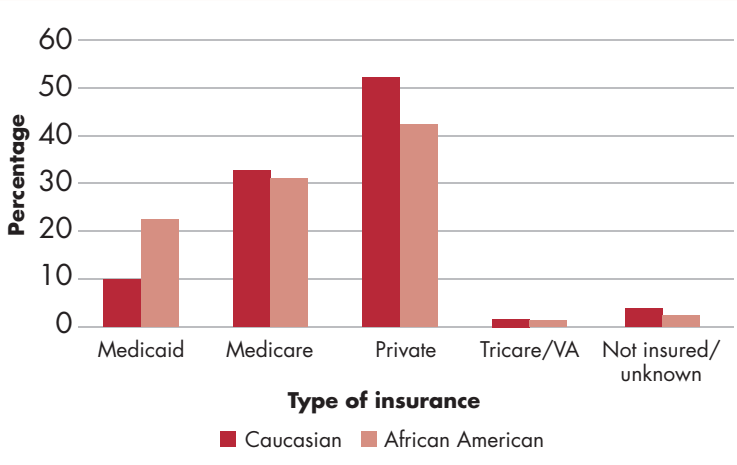

FIGURE 1 Insurance coverage by race for women diagnosed with breast cancer from the tumor registry of the Cancer Life Center at Navicent Health, Macon, Georgia, 2011 -2013. Tricare/VA, insurance coverage for military personnel and veterans under the Veterans Affairs

\section{Discussion}

Our study had some interesting findings. There was a statistically significant difference between African Americans and whites when it came to the clinical staging of breast cancer diagnosis. Whites were more often diagnosed as clinical stage I, whereas a greater percentage of African Americans were diagnosed at stages II, III, or IV. In addition, African Americans were more than twice as likely to be diagnosed with clinical stage IV breast cancer. These findings confirm a racial disparity in regard to breast cancer diagnosis. The etiology of these findings may prove to be ing mammograms commencing at the age of 40 years in the general population. ${ }^{13}$ The American Cancer Society recently updated its stance and now recommends routine mammograms starting at age 45 . Women aged 40-44 years should have a choice in screening as per the new recommendations. ${ }^{14}$

Socioeconomic factors, living in rural areas, and being African American are all risk factors for not pursuing screening mammograms. ${ }^{15}$ Mammography use among African American women seems to correlate with household income. ${ }^{3,16,17}$ This study demonstrated that white women with breast cancer in Central Georgia were more likely to have private insurance than were African American women with breast cancer. In addition, Medicaid rates among African Americans were significantly higher than among their white counterparts. These health insurance plans imply that the African American patients in this study may have been more likely to have lower socioeconomic status and level of education than were white women. This study did not assess screening mammography, but the insurance findings could suggest that the African Americans in our population may not have had easy access to mammography screening. ${ }^{18,19}$

Another crucial factor improving mammogram usage rates is the presence of a personal primary care physician (PCP) who can make recommendations that directly lead to higher use of medical services, such as screening mam- 
mography. Again, our study did not determine whether or not our patients had a $\mathrm{PCP}$ at the time of their cancer diagnosis. However, low-income individuals are less likely to have a PCP or regular access to health care and therefore may be postulated to be less likely to obtain screening mammography. ${ }^{20,21}$ Early breast cancer (stage 0 or I) is less likely to be palpable on clinical examination. Often times, these women are unaware and unsuspicious of the presence of breast cancer. Documenting these early-stage cancers is crucial, and screening mammography is postulated to be an attributed means of discovering it. Delays in screening mammography in these women can lead to continued tumor proliferation and spread, permitting cancer diagnosis to be at an advanced stage. Again, the possibility of delaying screening mammograms in our population was not assessed, but is implied based on insurance statuses. The data conveyed suggests that delays in access to screening mammograms are another possible explanation for why African American women are more likely to be diagnosed with a later clinical stage than are white women (Figure 2, Table 2).

\section{Biological factors}

Biological factors could also play a role in the disparities in breast cancer diagnosis and treatment between African American and white women. Breast cancer in younger women is more common in African Americans compared with their white counterparts. In addition, African American women tend to have more aggressive breast cancers at an earlier onset. ${ }^{22,23}$ For such women, the breast cancer diagnosis may be delayed because guidelines for screening mammography do not vary between the races. Race is not considered to be an independent risk factor and without additional risk factors, screening mammograms are not recommended before the age of 40 . It is possible that screening guidelines need to be reevaluated when it comes to racial discrepancies in the diagnosis of breast cancer.

Because there are differences by race and ethnicity in staging, treatment, and mortality rate, race and other biological factors should be addressed more aggressively in an attempt to improve overall breast cancer survival. ${ }^{24}$ Findings in this cohort also show a statistical significance in age of diagnosis. Of note is that African American women are diagnosed at a younger age and with a markedly advanced disease. These findings further suggest that the disease in African American women is different from

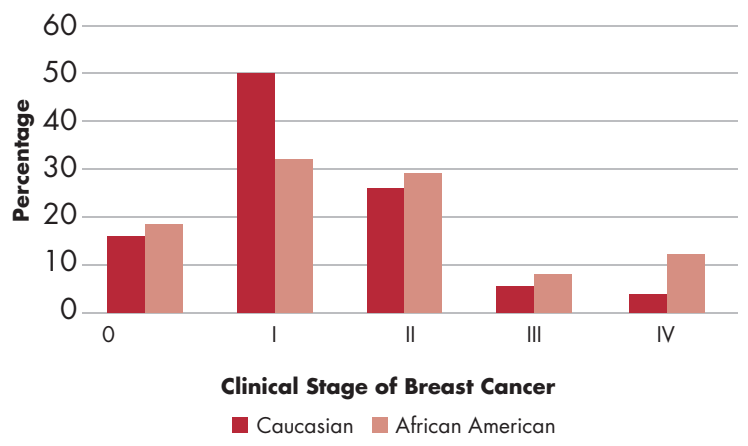

FIGURE 2 Clinical stage of cancer diagnosis in women diagnosed with breast cancer from the tumor registry of the Cancer Life Center at Navicent Health, Macon, Georgia, 201 1-2013.

what it is in white women. It can be postulated that African American women have a more aggressive disease, which may be attributable to biological factors.

\section{Health care provider}

White women are more likely to have a documented family history of breast cancer. ${ }^{25}$ It is difficult to say what the reason for this is: are physicians not asking their African American patients about risk factors and making appropriate recommendations, or are African American patients less likely to know or divulge their personal risk factors? The presence of risk factors changes the physician's approach 
in regard to aggressiveness with breast cancer screening. Physicians are an incredible resource to patients. Even simple teaching sessions by physicians about the importance of self-examinations for breast cancer are helpful. One study found that women who perform self-examinations are more likely to have a mammogram. ${ }^{26}$ If physicians are too busy or overwhelmed when it comes to assessing risk factors or teaching patient education, then perhaps surrogate systems, such as a patient navigator, may have some benefit in encouraging patient screening and follow-up. However, further studies would be needed in that regard, because the current system is usually arranged for postdiagnosis cancer care and not necessarily for screening purposes. ${ }^{4,27}$

There does seem to be a degree of mistrust of health care providers among the African American population. It is possible that this mistrust can be traced in part to the 1932-1972 Tuskegee Syphilis Study conducted in Alabama by the US Public Health Service among African American sharecroppers who had syphilis. The investigators were studying the natural progression of the disease, but the participants were told they were receiving free health care from the government. Even when penicillin became available and was the recognized and accepted mode of treatment, the men were prevented from taking it, leading to a number of deaths. ${ }^{28,29}$

A disparity in the race of health care providers may play an additional role in systemic mistrust. ${ }^{27}$ African Americans rate their patient care appointments with their physicians as less participatory than do whites. When patients see physicians of their own race, the patient participation rates increase. Improving interracial communication could be one means of overcoming mistrust of the physician. Likewise, increasing diversity among physicians may lead to improved patient care and participation among minority patients. ${ }^{30}$

\section{Limitations}

This study was a single-center, retrospective review and data analytics were limited to data entered into the tumor registry. It only examined patients in Central Georgia, so it is unclear if these findings would be translatable to other regions or locations. In addition, our study explores associations and not causality. Although we can state that there was a statistically significant difference in clinical staging along racial lines, we cannot state the reason with certainty. We could not assess what other factors might have played a role in the findings of this study. Socioeconomic status was not fully addressed. We could only assess insurance coverage, so it is difficult to say whether existing differences in clinical staging between the races are owing to true racial disparities as opposed to socioeconomic disparities. Nevertheless, the insurance status of our patients is noteworthy and it may be a good indicator for socioeco- nomic status. We also did not have information on biological factors or whether patients had primary care providers before their cancer diagnosis. However, again, health insurance status is a good indicator for socioeconomic status, which may be a good indicator for whether or not a patient had a PCP. In addition, the results in our study were still statistically significant in regard to disparities in clinical stage between the races, even when accounting for the unknown data. Our study shows that there are racial disparities between African American and white women in Central Georgia when assessing breast cancer diagnoses.

\section{Conclusion}

We examined racial disparities in breast cancer diagnosis between African American and white women in Central Georgia during 2011-2013. We found that African American women were more likely to be diagnosed with a later stage of breast cancer than were white women. They were also more likely to have Medicaid than private insurance. The etiology of these disparities is complex and multifaceted. Additional studies are needed to determine the true cause of these racial disparities, including socioeconomic, educational and biological roles, in order to eradicate these differences.

\section{References}

1. DeSantis C, Ma J, Bryan L, Jemal A. Breast cancer statistics, 2013. CA Cancer J Clin. 2014;64:52-62.

2. Li CL. Racial and ethnic disparities in breast cancer stage, treatment, and survival in the United States. Ethn Dis. 2005;15(suppl 2):S5-S9.

3. Patel K, Kanu M, Liu J, et al. Factors influencing breast cancer screening in low-income African Americans in Tennessee. J Comm Health. 2014;39:943-950.

4. Blackman DJ, Masi CM. Racial and ethnic disparities in breast cancer mortality: are we doing enough to address the root causes? J Clin Oncol. 2006;24:2170-2178.

5. Ries LAG, Eisner MP, Kosary CL, et al. Cancer Statistics Review, 1975-2002. Bethesda, MD, National Cancer Institute, 2005.

6. DeSantis CE, Fedewa SA, Goding Sauer A et al. Breast cancer statistics, 2015: Convergence of incidence rates between black and white women. CA Cancer J Clin. 2015 Oct 29 [Epub ahead of print]

7. Cui Y, Peterson N, Hargreaves M, et al. Mammography use in the Southern Community Cohort Study (United States). J Health Care Poor Underserved. 2007;18:102-117.

8. Guvenc I, Guvenc G, Tastan S, et al. Identifying women's knowledge about risk factors of breast cancer and reasons for having mammography. Asian Pacific J Cancer Preven. 2012;13:4191-4197.

9. Joan RB, Kyle G, Felicia H, et al. Factors affecting the use of screening mammography among African American Women. Cancer Epidemiology, Biomarkers and Prevention. 1991;1:75-82.

10. Kempe K, Larson R, Shetterley S, et al. Breast cancer screening in an insured population: whom are we missing? Perm J. 2013;17:38-44.

11. Sassi F, Luft H, Guadagnoli E, et al. Reducing racial/ethnic disparities in female breast cancer: screening rates and stages at diagnosis. Am J Public Health. 2006;96:2165-2172.

12. Smith RA, Duffy SW, Gabe R, Tabar L, Yen AM, Chen TH. The randomized trials of breast cancer screening: what have we learned? Radiol Clin North Am. 2004;42:793-806.

13. Gucalp A, Gaorav GP, Pilewsie ML, Sutton EJ, Norton L. Advances in managing breast cancer: a clinical update. F1000Prime Rep. 2014; 6:66.

14. Oeffinger KC, Fontham ET, Etzioni R et al. Breast Cancer Screen- 
ing for Women at Average Risk: 2015 Guideline Update From the American Cancer Society. JAMA. 2015 Oct 20;314(15):1599-614

15. Calle EE, Flanders WD, Thun MJ, Martin LM. Demographic predictors of mammography and pap smear screening in US women. American Journal of Public Health. 1993;83(1):53-60.

16. Lawson HW, Henson R, Bobo JK, et al. Implementing recommendations for the early detection of breast cancer and cervical cancer among low-income women. MMWR Recomm Rep. 2000;49(RR2):37-55

17. Vona-Davis L, Rose DP. The influence of socioeconomic disparities on breast cancer tumor biology and prognosis: a review. J Womens Health (Larchmt). 2009;18:883-893.

18. Breen N, Kessler L. Changes in the use of screening mammography: evidence from the 1987 and 1990 National Health Interview Surveys. Am J Public Health. 1994;84:62-67.

19. Rao RS, Graubard BI, Breen N, Gastwirth JL. Understanding the factors underlying disparities in cancer screening rates using the Peters-Belson approach: results from the 1998 national health interview survey. Med Care. 2004;42:789-800.

20. O'Malley MS, Earp JA, Hawley ST, Schell MJ, Mathews HF, Mitchell J. The association of race/ethnicity, socioeconomic status, and physician recommendation for mammography: who gets the message about breast cancer screening? Am J Public Health. 2001;91:49-54.

21. O'Malley AS, Forrest CB, Mandelblatt J. Adherence of low-income women to cancer screening recommendations. J Gen Intern Med. 2002;17:144-154.
22. Aziz H, Hussain F, Sohn C, et al. Early onset of breast carcinoma in African American women with poor prognostic factors. Am J Clin Oncol. 1999;22:436-440.

23. Newman LA, Alfonso AE. Age-related differences in breast cancer stage at diagnosis between black and white patients in an urban community hospital. Ann Surg Oncol. 1997;4:655-662.

24. Li CI, Malone KE, Daling JR. Differences in breast cancer stage, treatment, and survival by race and ethnicity. Arch Intern Med. 2003;163:49-56.

25. Murff HJ, Byrne D, Haas JS, Puopolo AL, Brennan TA. Race and family history assessment for breast cancer. J Gen Intern Med. 2005;20:75-80.

26. Bloom JR, Grazier K, Hodge F, Hayes WA. Factors affecting the use of screening mammography among African American women. Cancer Epidemiol Biomarkers Prev. 1991;1:75-82.

27. Dohan D, Schrag D. Using navigators to improve care of underserved patients. Cancer. 2005;104:848-855.

28. Freimuth VS, Crouse Quinn S, Thomas SB, Cole G, Zook E, Duncan T. African Americans' views on research and the Tuskegee Syphilis study. Soc Sci Med. 2001;52:797-808.

29. Gerend MA, Pai M. Social determinants of black-white disparities in breast cancer mortality: a review. Cancer Epidemiol Biomarkers Prev. 2008;17:2913-2923.

30. Cooper-Patrick L, Gallo JJ, Gonzales JJ, et al. Race, gender, and partnership in the patient-physician relationship. JAMA. 1999;282:583589. 